\title{
THE ROLE OF THE LOCUS COERULEUS IN REGULATION OF SEIZURE SUSCEPTIBILITY IN RATS
}

\author{
Ryozo OISHI* and Noriko SUENAGA \\ Department of Pharmacology. Faculty of Medicine. Kysushu University. \\ Fukuoka 812, Japan
}

Accepted June 29, 1982

\begin{abstract}
When studying the role of the locus coeruleus (LC) in the regulation of seizure susceptibility in rats, we found that bilateral LC lesion significantly lowered the electroshock seizure threshold for the tonic extension of forelegs. The pattern of maximal electroshock seizure was not affected by LC lesion, although the recovery time was slightly prolonged. In the pentylenetetrazol (PTZ) seizure threshold test. LC lesion significantly elevated the twitch threshold, but did not affect the threshold for the generalized clonic seizure or clonic convulsion. Incidence of the tonic extension in the PTZ seizure test tended toward an increase with LC lesion. Biochemical determinations revealed that LC lesion significantly decreased the norepinephrine contents in various brain regions as well as the 5 hydroxytryptamine contents of some brain regions, but not the 5-hydroxyindoleacetic acid contents. These results suggest that while the $L C$ is involved in the regulation of seizure susceptibility, the involvement differs with various types of seizures.
\end{abstract}

There is now considerable evidence that central monoamines are involved in the regulation of susceptibility to various seizures (1). Kilian and Frey (2) observed that dopamine - $\beta$ - hydroxylase inhibitors, disulfiram and $F L A-63$, increased the seizure susceptibility for electroshock or pentylenetetrazol (PTZ) as well as $\alpha$-mentyltyrosine. Furthermore, the reduction of brain catecholamine by intraventricularly injected 6hydroxydopamine (6-OHDA) lowered the electroshock $(3,4)$ and PTZ seizure thresholds $(5,6)$ and enhanced the duration and number of PTZ seizures (7). The effect of 6-OHDA on the electroshock threshold was diminished by treatment with desipramine (4). which inhibits the uptake of 6-OHDA into

*Present address: Department of Pharmacology, Okayama University Medical School, Okayama 700. Japan the norepinephrine (NE) neurons but not into the DA neurons. These results suggest that NE plays an essential role in the regulation of seizure susceptibility, although 5-hydroxytryptamine (5-HT) also contributes to the regulation of the occurrence of seizures (2).

The locus coeruleus (LC) is the largest cell group of the central NE neurons and innervates the cortex, hippocampus, cerebellum. spinal cords and many other areas $(8,9)$. Studies have been done on the effect of dorsal bundle lesion on seizure susceptibility (5. 10, 11). However, there is little documentation of changes in seizure susceptibility in LC lesioned animals. Therefore, we examined the influence of LC lesion on various seizures in an attempt to clarify the integrated role of the $L C$ in the regulation of seizure susceptibility. In addition, when the rats were 
decapitated for biochemical assays, a postdecapitation convulsion was also measured since the inhibition of the decapitation convulsion seems to be a good index to assess the extent of LC lesion (12).

\section{MATERIALS AND METHODS}

Electrolytic lesion of bilateral locus coeruleus: Adult male Wistar King A rats from the Kyushu University Institute of Laboratory Animals, weighing $200-220 \mathrm{~g}$. were anesthetized with pentobarbital sodium $(40 \mathrm{mg} /$ $\mathrm{kg}$. i.p.) and placed on a stereotaxic instrument according to the procedure of König and Klippel (13). An unipolar electrode made of stainless steel wire of $0.2 \mathrm{~mm}$ in diameter. insulated except for the tip, was inserted into the LC (posterior $2.0 \mathrm{~mm}$, lateral $1.1 \mathrm{~mm}$. ventral $7.0 \mathrm{~mm}$ deep from the skull): and a $2 \mathrm{~mA}$ direct current was applied for $20 \mathrm{sec}$ in each side. In the control, a sham operation was performed in the same manner without applying electric current. The rats were housed in groups of 4 or 5 in a colony room maintained on a $12 \mathrm{hr}$ light/dark cycle and controlled at $22 \pm 2{ }^{\circ} \mathrm{C}$ thermostatically.

Electroshock seizure threshold: The electroshock seizure threshold, that is, the current necessary to produce a tonic extension of the forelegs, was determined by the "up and down" method described by Kimball et al. (14). Seizure was induced by stimulation with $60 \mathrm{~Hz}, 0.2 \mathrm{sec}$ in duration, through ear clip electrodes. The current was varied every $1 \mathrm{~mA}$ between 19 and $23 \mathrm{~mA}$.

Maximal electroshock seizure: Maximal electroshock seizure was induced by a supramaximal current of $150 \mathrm{~mA}, 60 \mathrm{~Hz}, 0.2$ $\mathrm{sec}$ in duration through ear clip electrodes. The durations of tonic flexion, tonic extension of forelegs, tonic extension of hindlegs, and recovery time (total time to recover the righting reflex) were measured.

Pentylenetetrazol seizure threshold: The rats were briefly restrained during an infusion of a $5 \%$ solution of PTZ (Sigma) into a lateral tail vein at a constant rate of $0.004 \mathrm{ml} / \mathrm{sec}$ using a syringe pump (Harvard Apparatus 975). Durations of infusion required to elicit the initial twitch, generalized clonic seizure, and final clonic convulsion were measured. The seizure pattern by intraveous infusion of PTZ was described in detail in our previous report (5). After the test, each rat was given $5 \mathrm{mg} / \mathrm{kg}$ diazepam (Yamanouchi), i.v., to protect against death from possible overdosing of PTZ.

Post-decapitation convulsion, biochemical determinations and histology: The rats were decapitated 28 days after surgery, and the latency and duration of decapitation convulsions were measured.

The brain was immediately removed, placed on ice and separated into 4 parts, that is, the telencephalon, diencephalon, brainstem and cerebellum. The spinal cord (thoracic and lumbar regions) was also removed. Half samples of each group were homogenized in $0.4 \mathrm{~N}$ perchloric acid containing $0.1 \%$ sodium metabisulfite and $0.005 \%$ disodium EDTA, and the NE contents were assayed by the method of Shellenberger and Gordon (15). The remaining samples in each group were homogenized in $0.1 \mathrm{~N} \mathrm{HCl}$ containing $0.1 \%$ cysteine. 5- HT was determined by the method of Snyder et al. (16), and 5-HIAA was purified by the method of Giacalone and Valzelli (17) and determined by the OPT method described by Curzon and Green (18).

If a rat died during the experimental period. the brain was quickly removed and placed in a $10 \%$ formalin solution. After several days. these brains were sectioned at a thickness of $50 \mathrm{\mu m}$ on a freezing microtome, and the sections were stained with cresyl violet. Inadequately lesioned tissues were discarded.

Statistical evaluations: Statistical evaluations of experimental data were performed using the two-tailed Student's t-test, unless otherwise described. The convulsive current 
50 (CC50) in the experimental group was considered to be significantly different from the control CC50 if it lay outside the $95 \%$ confidence limit of the latter and also the control CC50 lay outside the $95 \%$ confidence limit of the experimental CC50 (19).

\section{RESULTS}

Electroshock seizure threshold: Rats were subjected to the electroshock seizure threshold test 10 days after the LC lesion. As shown in Fig. 1, the incidence of tonic extension of forelegs was greater in the LC lesioned group, and the seizure threshold was significantly lowered as compared with that of the control.

Maximal electroshock seizure: Rats were given a maximal electroshock 14 days after the LC lesion. Both control and LC lesioned groups showed a similar sequence of convulsions, that is, about $1 \mathrm{sec}$ of tonic flexion and $15 \mathrm{sec}$ of tonic extension of the forelegs. which included $5 \mathrm{sec}$ of complete tonic extension of hindlegs. However, the LC lesioned group showed a slightly prolonged recovery time, as compared with control, although there was not a statistically significant difference $(t=2.01, P<0.1)$. No rat of either group died after convulsions induced in our tests.

Pentylenetetrazol seizure threshold: The PTZ seizure threshold test was performed 21 days after the LC lesion. Continuous injection of PTZ into a lateral tail vein of rats produced a few initial twitches, a generalized clonic seizure accompanied by a loss of righting reflex, and then myoclonic jerks leading to the tonic extension of the forelegs in some cases, and finally clonic convulsion.

As shown in Table 1. LC lesion significantly elevated the threshold for the twitch $(P<0.05)$, but did not change those for the generalized clonic seizure and clonic convulsion. The LC lesioned group showed a higher incidence of tonic extension of the forelegs, although there was not a significant difference $(P=0.067$. Fisher's exactly probability test).

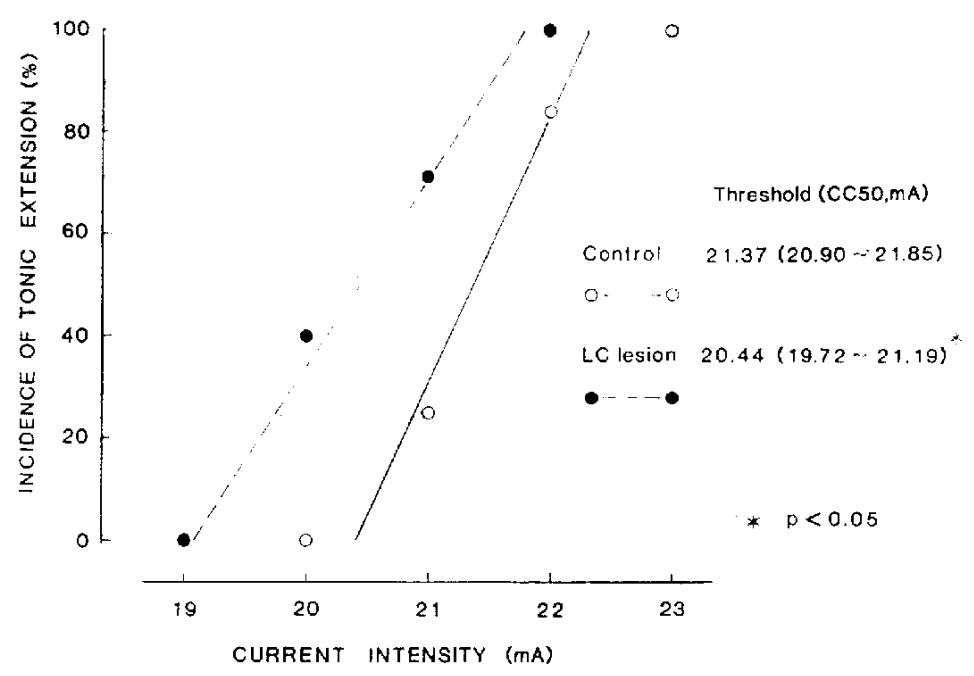

Fig. 1. Effect of bilateral lesion of the locus coeruleus (LC) on the electroshock seizure threshold. Threshold for producing the tonic extension of forelegs is expressed as $50 \%$ of the convulsive current ( $\mathrm{CC50}$ ) in $\mathrm{mA}$ with $95 \%$ confidence intervals calculated by the "up and down" method of Kimball et al. (14). Total numbers of rats are 17 and 16 in the control and LC lesioned groups, respectively. 
Table 1. Effect of bilateral lesion of the locus coeruleus (LC) on the pentylenetetrazol (PTZ)

\begin{tabular}{lcc}
\hline & Control & LC lesion \\
\hline Number of rats & 16 & 12 \\
Threshold $(\mathrm{mg} / \mathrm{kg})$ & & \\
$\quad$ Twitch & $31.31 \pm 0.85$ & $35.84 \pm 1.74^{*}$ \\
$\quad$ Generalized clonic seizure & $40.04 \pm 0.81$ & $42.49 \pm 1.61$ \\
$\quad$ Clonic convulsion & $72.33 \pm 1.79$ & $72.07 \pm 2.08$ \\
$\quad$ The incidence of tonic extension & $0 / 16$ & $3 / 12$ \\
\hline
\end{tabular}

Each threshold was determined by the constant infusion of $5 \% \mathrm{PTZ}$ into a lateral tail vein and expressed as the mean \pm S.E.M. dose of PTZ per $\mathrm{kg}$ of body weight. $\cdot P<0.05$, compared with the control group.

Table 2. Post-decapitation convulsion 28 days after bilateral lesion of the locus coeruleus (LC)

\begin{tabular}{lcc}
\hline & Control & LC lesion \\
\hline Number of rats & 17 & 10 \\
Latency (sec) & $2.4 \pm 0.1$ & $14.3 \pm 0.4^{*}$ \\
Duration (sec) & $18.1 \pm 0.4$ & $9.3 \pm 0.5^{*}$ \\
\hline
\end{tabular}

Each value is expressed as the mean \pm S.E.M. ${ }^{*} P<0.001$, compared with the control group.

Post-decapitation convulsion: All rats were decapitated 28 days after the $\mathrm{LC}$ lesion. In the control rats, the decapitation convulsion generally included clonic convulsions of the hindlegs with a latency of $2.4 \mathrm{sec}$ on the average and lasted for $18.1 \mathrm{sec}$. The LC lesion markedly prolonged the latency and shortend the duration of the very weak convulsion (Table 2).

Biochemical determinations: Table 3 shows the NE, 5-HT and 5-HIAA contents in various brain regions 28 days after the surgery. The NE contents of all regions were significantly decreased by LC lesion. The 5-HT contents were significantly decreased in the diencephalon, cerebellum and the spinal cord. Conversely, the 5-HIAA content was significantly increased in only the telencephalon. No significant differences were seen in the other regions.

\section{DISCUSSION}

We attempted to clarify the integrated role of the $L C$ in the regulation of seizure susceptibility. The LC lesion lowered the electroshock seizure threshold, and there was a slightly higher incidence of the tonic extension in the PTZ seizure test as compared to findings in the controls. However, the potentiation by LC lesion was not apparent in the case of maximal electroshock seizure or the PTZ seizure threshold test. These results suggest that the involvement of the LC in the regulation of seizure susceptibility differs with various seizure tests.

Bilateral LC lesion significantly decreased the NE contents of all areas of the brain. In the cortex, the NE content was decreased to $29 \%$ of the control value. This suggests that the LC lesion in our experiment extended to more 
Table 3. Contents of norepinephrine (NE), 5-hydroxytryptamine (5-HT) and 5-hydroxyindoleacetic acid (5-HIAA) in varlous regions of the rat central nervous system 28 days after bilateral lesion of the locus coeruleus (LC)

\begin{tabular}{lllr}
\hline & Control & LC lesion & $\%$ \\
\hline NE & $0.21 \pm 0.01$ & $0.06 \pm 0.01^{\cdots *}$ & 29 \\
Cortex & $0.23 \pm 0.01$ & $0.10 \pm 0.01^{\cdots *}$ & 43 \\
Telencephalon & $0.56 \pm 0.01$ & $0.38 \pm 0.03^{* *}$ & 68 \\
Diencephalon & $0.50 \pm 0.02$ & $0.30 \pm 0.01^{\cdots *}$ & 60 \\
Brainstem & $0.19 \pm 0.01$ & $0.04 \pm 0.01^{\cdots \cdots}$ & 21 \\
Cerebellum & $0.18 \pm 0.01$ & $0.08 \pm 0.01^{\cdots *}$ & 44 \\
Spinal cord & & & \\
5-HT & $0.53 \pm 0.01$ & $0.50 \pm 0.04$ & 94 \\
Telencephalon & $0.78 \pm 0.02$ & $0.72 \pm 0.01^{*}$ & 92 \\
Diencephalon & $0.85 \pm 0.02$ & $0.84 \pm 0.02$ & 99 \\
Brainstem & $0.24 \pm 0.01$ & $0.18 \pm 0.01^{\cdots \cdots}$ & 75 \\
Cerebellum & $0.55 \pm 0.01$ & $0.47 \pm 0.02^{* *}$ & 85 \\
Spinal cord & & & \\
5-HIAA & & $0.28 \pm 0.01^{*}$ & 112 \\
Telencephalon & $0.25 \pm 0.01$ & $0.65 \pm 0.01$ & 97 \\
Diencephalon & $0.67 \pm 0.01$ & $0.66 \pm 0.02$ & 80 \\
Brainstem & $0.62 \pm 0.02$ & $0.08 \pm 0.01$ & 95 \\
Cerebellum & $0.10 \pm 0.00$ & $0.21 \pm 0.01$ & 06 \\
Spinal cord & $0.22 \pm 0.01$ & & \\
\hline
\end{tabular}

Each value is expressed as the mean \pm S.E.M. $(\mu \mathrm{g} / \mathrm{g})$ of 4 to $7 \mathrm{rats} \%=$ the percentage of the mean values of the control group. $P<0.05,{ }^{*} P<0.01 .{ }^{* *} P<0.001$ as compared with the control group.

than $70 \%$ of the NE neurons of the LC, since the $L C$ is considered to serve as the exclusive source of NE neurons in the cerebral cortex $(9,20)$.

As there is reportedly an interaction between the NE and 5-HT systems in the brain (21). the 5-HT and 5-HIAA contents were also assayed in LC lesioned rats. The 5 -HT contents in some brain regions were significantly decreased after the lesion, in particular, that of the cerebellum was decreased to $75 \%$ of the control value. This may due to damage extending to the cerebellar peduncles near the LC. The 5-HIAA contents, however, were not significantly decreased in all regions, indicating that the function of the 5-HT system in the brain was not decreased after LC lesion.

Browning and Maynert (3) reported that the intracisternal 6-OHDA treatment caused a significant lowering of the electroshock seizure threshold as well as decreases in the NE and DA contents in the brain to about 30 and $35 \%$ of the control value, respectively. A great decrease in the threshold was reported by Quattrone et al. (4) in rats treated with both 6-OHDA and pargyline. Here the NE and DA contents in the brain were decreased to $20 \%$ of the control value. These workers excluded the involvement of the decrease in DA content in this phenomenon since the treatment with desipramine diminished the potent effect of 6-OHDA and pargyline. In the present study, the LC lesion significantly lowered the electroshock seizure threshold to about the same degree as that observed by Browning and Maynert (3). Thus, the LC may be the most important catecholamine- 
related system for the regulation of the electroshock seizure threshold.

It has been reported that 6-OHDA prolonged the duration of tonic extension induced by maximal electroshock in rats (3) and mice (22). The LC lesion, however, did not change the duration of tonic extension of either forelegs or hindlegs. In case of minimal electroshock seizure induced by $10 \mathrm{~mA}$, Mason and Corcoran (10) observed that the depletion of spinal NE elevated the incidence of tonic extension. However, we found no correlation between the spinal NE content and the duration of tonic extension in maximal electroshock seizure (23). In LC lesioned rats. there was a $15 \%$ longer recovery time, but this effect was much less than that observed in intraventricularly 6-OHDA treated rats (3) or mice (24). Furthermore, it is unlikely that the elevated 5-HT neuron activity in the telencephalon shown by the increase in the 5-HIAA content diminished the effect of LC lesion, since 5-hydroxytryptophan treatment reportedly causes a considerable prolongation of the recovery time (24).

We previously reported that the lesion of the dorsal or ventral NE bundle did not change the threshold amounts of PTZ required to elicit twitch or clonic convulsion; although these thresholds were significantly lowered by 6-OHDA (5), $\alpha$-methyltyrosine and $\mathrm{DBH}$ inhibitors (2). In the present experiment, LC lesion elevated the twitch threshold.

Interestingly, these findings are similar to observations in rats treated with 6-OHDA at 8 days after birth (5). These rats showed a marked depletion of NE in the brain except for the brainstem, in which the NE content was increased. All these findings taken together suggest that NE systems different from those in the $\mathrm{LC}$ are involved in the regulation of the PTZ seizure threshold, particularly in the brainstem region.
On the other hand. Mason and Corcoran (11) reported that the dorsal bundle lesion enhanced the duration and number of seizures and also the incidence of tonic extension after i.p. administration of $70 \mathrm{mg} /$ $\mathrm{kg}$ PTZ in rats. Similar results were obtained in rats treated with 6-OHDA intraventricularly (7). However, it should be noted that in these rats, the latency to the myoclonic jerk or convulsion was never shortened. In the present experiment, the LC lesioned rats also showed a slightly higher incidence of the tonic extension as compared with the controls. The tonic extension is considered as a stronger type than the clonic seizures. We also observed that 22 or $80 \%$ of rats treated with intraventricular 6-OHDA (150 $\mu \mathrm{g} \times 2)$ or reserpine (2 $\mathrm{mg} / \mathrm{kg}$, i.p.), respectively. showed tonic extension in the same PTZ seizure test as used in the present experiment (unpublished observation). Therefore, the LC may be involved in the regulation of the developed convulsion by PTZ to a much greater extent than the inhibition of the seizure occurrence itself.

The post-decapitation convulsion was strongly inhibited by LC lesion, in good agreement with our previous studies (12. 23. 25) in which the coeruleospinal NE neurons were found to play a critical role in the etiology of decapitation convulsion.

In conclusion, we confirmed that the LC system regulates the susceptibility to certain induced seizures, particularly the electroshock seizure threshold. However, the involvement of the LC in seizure regulation differs with various types of seizures.

Acknowledgements: We thank $M$. Ohara. Kyushu University. for comments on the manuscript.

\section{REFERENCES}

1) Maynert, E.W., Marczynski, T.J. and Browning. R.A.: The role of the neurotransmitters in the: epilepsies. Adv. Neurol. 13, 79-147 (1975) 
2) Kilian, M. and Frey, H.-H.: Central monoamines and convulsive thresholds in mice and rats. Neuropharmacology 12, 681-692 (1973)

3) Browning, R.A. and Maynert, E.W.: Effect of intracisternal 6-hydroxydopamine on seizure susceptibility in rats. Eur. J. Pharmacol. 50, 97-101 (1978)

4) Quattrone, A., Crunelli, V. and Samanin, R.: Seizure susceptibility and anticonvulsant activity of carbamazepine. diphenylhydantoin and phenobarbital in rats with selective depletions of brain monoamines. Neuropharmacology 17, 643-647 (1978)

5) Oishi, R., Suenaga, N. and Fukuda, T.: Possible involvement of brainstem norepinephrine in pentylenetetrazol convulsions in rats. Pharmacol. Biochem. Behav. 10, 57-61 (1979)

6) Oishi, R.. Suenaga, N., Hidaka, T. and Fukuda, T.: The role of $\alpha$-adrenoceptors in the regulation of pentylenetetrazol convulsions in mice. J. Pharm. Pharmacol. 31, 709-710 (1979)

7) Corcoran, M.E., Fibiger, H.C., McGeer, E.G. and Wada, J.A.: Potentiation of leptazol seizures by 6-hydroxydopamine. J. Pharm. Pharmacol. 25. 497-499 (1973)

8) Ungerstedt, $U$ :: Stereotaxic mapping of the monoamine pathways in the rat brain. Acta Physiol. Scand. Supp. 367, 1-48 (1971)

9) Lindvall, 0 . and Björklund. A.: The organization of the ascending catecholamine neuron systems in the brain. Acta Physiol. Scand. Supp. 412, 1-48 (1974)

10) Mason, S.T, and Corcoran, M.E.: Seizure susceptibility after depletion of spinal or cerebellar noradrenaine with 6-OHDA. Brain Res. 166, 418-421 (1979)

11) Mason, S.T. and Corcoran, M.E.: Catecholamines and convulsions. Brain Res. 170, 497507 (1979)

12) Suenaga, N., Oishi, R. and Fukuda, T.: The role of locus coeruleus in decapitation convulsions of rats. Brain Res. 177, 83-93 (1979)

13) König, J.F.R. and Klippel, R.A.: The Rat Brain. A Stereotaxic Atlas of the Forebrain and Lower Parts of the Brain Stem. Williams and Wilkins, Baltimore (1963)

14) Kimball, A.W., Burnett, W.T., Jr. and Doherty. D.G.: Chemical protection against ionizing radiation. I. Sampling methods for screening compounds in radiation protection studies with mice. Radiat. Res. 7, 1-12 (1957)
15) Shellenberger, M.K. anf Gordon, J.H.: A rapid simplified procedure for simultaneous assay of norepinephrine, dopamine and 5-hydroxytryptamine for discrete brain areas. Anal. Biochem. 39, 356-372 (1971)

16) Snyder, S.H., Axerlod, J. and Zweig, M.: A sensitive and specific fluorescence assay for tissue serotonin. Biochem. Pharmacol. 14, 831 $835(1965)$

17) Giacalone, E. and Valzelli, L.: A method for the determination of 5-hydroxyindole-3-acetic acid in brain. J. Neurochem. 13, 1265-1266 (1966)

18) Curzon, G. and Green, A.: Rapid method for the determination of 5-hydroxytryptamine and 5 hydroxyindoleacetic acid in small regions of rat brain. Br. J. Pharmacol. 39, 653-655 (1970)

19) Wong, C.L. and Wai, M.K.: The effect of prostagrandin $E_{2}$ on increased naloxone potency induced by morphine treatment. Eur. J. Pharmacol. 74, 365-367 (1981)

20) Maeda, T., Pin, C., Salvert, D., Ligier, M. and Jouvet, $M$.: Les neurones contenant des catécholamines du tegmentum pontique et leurs voies de projection chez le chat. Brain Res. 57, 119-152 (1973)

21) Kostowski, W., Samanin, R., Bareggi, S.R., Marc, V., Garattini, S. and Valzelli, L.: Biochemical aspects of the interaction between midbrain raphe and locus coeruleus in the rat. Brain Res. 82, 178-182 (1974)

22) Fukuda, T., Araki, Y. and Suenaga, N.: Inhibitory effects of 6 -hydroxydopamine on the clonic convulsions induced by electroshock and decapitation. Neuropharmacology 14, 597-583 (1975)

23) Oishi, R., Suenaga, N., Hidaka, T. and Fukuda, T.: Inhibitory effect of intraspinal injection of 6 -hydroxydopamine on the clonic convulsion in maximal electroshock seizure. Brain Res. 169 , 189-193 (1979)

24) Fukuda, T., Araki, Y., Takishita, S. and Koga, T.: Correlation between brain monoamine levels and postictal coma following electroshock. Arch. Int. Pharmacodyn. Ther. 213, 58-63 (1975)

25) Suenaga, N., Yamada, K. and Fukuda, T.: Correlation between central catecholamine level and post-decapitation convulsion in rats treated with 6-hydroxydopamine. Brain Res. $122,165-169$ (1977) 